\title{
ECHOCARDIOGRAPHIC DETERMINANTS FOR DIASTOLIC DYSFUNCTION IN CHRONIC KIDNEY DISEASE
}

\author{
Smit Shrivastava1 ${ }^{1}$ Suresh Chandravanshi², Prakash Sultania ${ }^{3}$, Abhikrishna Singh ${ }^{4}$, Sunil Kumar Karna ${ }^{5}$, Rimjhim Shrivastava ${ }^{6}$ \\ ${ }_{1}^{1}$ Associate Professor and HOD, Department of Cardiology, Advanced Cardiac Institute, Pt JNM Medical College, Raipur, Chhattisgarh, \\ India. \\ ${ }^{2}$ Associate Professor, Department of Medicine, Pt JNM Medical College, Raipur, Chhattisgarh, India. \\ ${ }^{3}$ Resident Medical Officer, Department of Medicine, Pt JNM Medical College, Raipur, Chhattisgarh, India. \\ ${ }^{4}$ Clinical Associate, Department of Cardiology, Cardiology Clinic, Raipur, Chhattisgarh, India. \\ ${ }^{5}$ Cardiologist, Department of Cardiology, BM Petal Cardiac Centre, Sri Krishna Hospital, Karamsad, Gujarat, India. \\ ${ }^{6}$ Attending Physician, Cardiology Clinic, Raipur, Chhattisgarh, India.
}

\section{BACKGROUND}

ABSTRACT

Diastolic dysfunction is associated with adverse outcome. There are no direct means to determine diastolic function by echocardiography, however, a multitude of echocardiography parameters assist in determining the diastolic dysfunction. The application of these parameters is not well validated in chronic kidney disease subjects.

\section{MATERIALS AND METHODS}

This study used echocardiography parameters to determine diastolic function in chronic kidney disease and in normal subjects.

\section{RESULTS}

Left atrial volume index appears to be a strong parameter for determining diastolic dysfunction in this subset.

\section{CONCLUSION}

Echocardiographic parameters as a standalone tool for determining diastolic dysfunction in the presence of chronic kidney disease are inadequate. Therefore, a combination of these parameters becomes mandatory to gauge the diastolic dysfunction in chronic kidney disease patients. Left atrial volume index may be proposed as a significant tool for diastolic dysfunction and it should be an essential part of echocardiographic interrogation in chronic kidney disease patients.

\section{KEY WORDS}

Diastolic Dysfunction, Chronic Kidney Disease

HOW TO CITE THIS ARTICLE: Shrivastava S, Chandravanshi S, Sultania P, et al. Echocardiographic determinants for diastolic dysfunction in chronic kidney disease. J. Evolution Med. Dent. Sci. 2019;8(12):927-929, DOI: 10.14260/jemds/2019/206

\section{BACKGROUND}

Left ventricular (LV) diastolic function can be quantified by invasive and non-invasive methods. The invasive methods for quantifying the diastolic function include the peak instantaneous rate of $\mathrm{LV}$ pressure decline $(-\mathrm{dP} / \mathrm{dt})$, the time constant of LV relaxation (tau), and the stiffness modulus. Although, these parameters cannot be directly quantified by echocardiography examination, echocardiography remains the most pragmatic clinical method for evaluating LV diastolic function based on its omnipresence and effortless applicability. As the diastolic properties of LV is determined the congeneric contribution of left atrial (LA) phasic function to LV filling. In subjects with normal diastolic function, the relative contribution of the reservoir, conduit and contractile function of the LA to the filling of LV is approximately $40 \%$, $35 \%$ and $25 \%$ respectively.

As relaxation mechanics of $\mathrm{LV}$ deteriorates, the comparative contribution of LA reservoir and contractile

'Financial or Other Competing Interest': None.

Submission 22-12-2018, Peer Review 08-03-2019,

Acceptance 16-03-2019, Published 25-03-2019.

Corresponding Author:

Dr. Smit Shrivastava,

Head, Department of Cardiology,

Advanced Cardiac Institute,

PtJNM Medical College, Raipur, Chhattisgarh, India.

E-mail: dr.smit.shrivastava@gmail.com

DOI: $10.14260 /$ jemds $/ 2019 / 206$

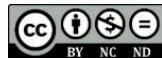

function escalates while passive conduit function dominates. Eventually the LA functions as a conduit between pulmonary veins and LV as advanced diastolic dysfunction develops.(1)

The left atrial volume is a barometer of left ventricular filling pressure and reflects the severity of the left ventricular diastolic function. The determinants of left atrial volume are primarily: the left ventricular filling pressure and anthropometry determinants, age, atrial fibrillation and chronic volume overload.(2) Beyond the childhood years, the left atrial volume index (LAVI) becomes independent of age variable(3) The age related LA enlargement mirrors the pathophysiological changes that accompany advancing age rather than a consequence of chronological aging. It is clear from epidemiological studies that the leading risk factors for diastolic dysfunction are older age, systemic hypertension, chronic kidney disease, obesity and sedentary lifestyle, and myocardial ischemia.

This study aims at ascertaining the optimum echocardiographic parameter for determining the diastolic dysfunction caused by chronic kidney disease in reduced ejection patients resulting from coexisting myocardial infarction.

\section{MATERIALS AND METHODS \\ Study Design}

Cross Sectional Observational study. 
This study was initiated after obtaining institutional ethics committee approval for the protocol. This was a hospital based cross sectional observational study, conducted in Department of Medicine PT J N M Medical College and Dr. B R A M Hospital between July 2014 to December 2014 Raipur in patient with history of anterior wall myocardial infarction resulting in ejection fraction less than 45 percent. All the patients who satisfied the study inclusion criteria between the time frame mentioned above and who gave written informed consent were included into the study. The echocardiographic parameters for quantifying diastolic dysfunction - mitral inflow velocities (MVE and MVA), deceleration time (DT) and LA volume were determined in the two cohorts of 50 subjects in the study, one with documented chronic kidney disease and other without kidney disease respectively.

\section{Assessment of LA Size}

LA is asymmetrical complex three-dimensional anatomical structure. Furthermore, LA enlargement may not proceed in a uniform and stipulated manner. Therefore, anteroposterior measurement of LA by M-mode echocardiography is likely to confer an insensitive assessment of any change in LA size. On the contrary, LA volume estimated by echocardiography provides a more accurate and reproducible appraisal of LA size when compared to magnetic resonance imaging (MRI) and cine-computerized tomography (CT).

The LA size was measured at the ventricular end-systole when the LA chamber is at its superlative proportion. The pulmonary vein confluence and LA appendage are purposefully omitted during planimetry interrogation. Echocardiographic assessment of LA volume was done by area -length method.

\section{Biplane Area- Length Method}

Orthogonal apical views, apical four and two-chamber views were procured for quantifying of LA area and length. The length was determined from the midpoint at the plane of mitral annulus to posterior wall. Left atrial volume was then calculated on the basis of the equation LA volume $=0.85 \times$ $(A 1 \times A 2) \div \mathrm{L}$; where $\mathrm{A} 1$ and $\mathrm{A} 2$ were the areas of LA in four and two chamber views and $L$ was the shortest of the lengths obtained from the orthogonal views and indexed to body surface area. This method has been recommended by the ASE guidelines for estimation of LA volume. (Lang RM et al 2005).

The LAVI (left atrial volume index) was obtained after indexing upon the body surface area for the subject.

Statistical analysis Data was entered into Microsoft excels worksheet and then imported into SPSS (IBM Corp. Released 2012. IBM SPSS Statistics for Windows, Version 21.0. Armonk, NY: IBM Corp) for analysis. Data were presented as mean + SD. Shapiro-Wilk test was used to test for normalcy of data. Mann-Whitney U test was used for comparison of data that were not normally distributed and independent samples t-test was used to compare data for normally distributed variables. A P-value $<0.05$ was considered statistically significant.

The table 1 shows the comparison of echocardiographic diastolic dysfunction parameters between chronic kidney disease and normal renal function subjects.

\section{RESULTS}

This study revealed that the usual echocardiographic determinants of diastolic dysfunction in the form of mitral inflow velocities and their ratio and the deceleration time were not evidently different between the two groups of chronic kidney disease and normal kidney function. However, the left atrial volume index was significantly higher $(\mathrm{p}<0.0001)$ in chronic kidney disease group as compared to the normal kidney function group. This finding suggests that the left atrial volume index maybe the optimum echocardiographic determinant of diastolic dysfunction assessment in patients with chronic kidney disease who have systolic dysfunction due to myocardial infarction.

\begin{tabular}{|c|c|c|c|c|c|c|}
\hline \multirow{2}{*}{ Variables } & \multicolumn{2}{|c|}{$\begin{array}{c}\text { CKD (21 } \\
\text { Patients) }\end{array}$} & \multicolumn{2}{c|}{$\begin{array}{c}\text { NON-CKD(29 } \\
\text { Patients) }\end{array}$} & $\begin{array}{c}\text { Mann- } \\
\text { Whitney } \\
\text { U }\end{array}$ & $\begin{array}{c}\text { p- } \\
\text { Value }\end{array}$ \\
\cline { 2 - 7 } & Mean & S.D. & Mean & S.D. & 184.500 & 0.016 \\
\hline Age & 68.57 & 8.304 & 62.83 & 3.083 & 184.500 & 0.752 \\
\hline MVE & 69.25 & 14.266 & 68.49 & 15.021 & 288.500 & 0.752 \\
\hline MVA & 73.78 & 23.222 & 76.38 & 18.151 & 303.000 & 0.976 \\
\hline MVA/MVE & 0.94 & 12.72 & 0.89 & 11.73 & 295.75 & 0.864 \\
\hline DT & 243.14 & 69.183 & 255.79 & 103.080 & 290.000 & 0.775 \\
\hline EF & 32.38 & 6.778 & 37.52 & 6.039 & 185.500 & 0.017 \\
\hline LAVI & 36.24 & 5.403 & 28.38 & 5.185 & 93.500 & 0.0001 \\
\hline BMI & 24.07 & 2.823 & 25.87 & 1.339 & 220.000 & 0.097 \\
\hline BSA & 1.53 & 0.141 & 1.62 & 0.118 & 182.000 & 0.016 \\
\hline \multicolumn{6}{|c|}{ Table 1 } \\
\hline
\end{tabular}

\section{DISCUSSION}

The CASCADE study(4) concluded that the diastolic function of LV was best determined by a combination of echocardiographic parameters namely Em, E/m ratio and LAVI rather than any single parameter. Mads Ersboll, Mads J. Anderson et al 2012(5) did prospective study on 843 pt of acute myocardial infarction within one week of MI with mean age of $62.1 \pm 11$.8yr where $74 \%$ was male. There was positive correlation seen among LAVI and age, while there was negative correlation between EF and LAVI.

Similarly, Leia Hee, Tuan Nguyen, et al 2014,(6) studied 289 unselected consecutive patients to compare stage 3 or 4 CKD ( $\mathrm{n}=49$ ) patients with those with eGFR $>60 \mathrm{ml} / \mathrm{min}$ per $1.73 \mathrm{~m} 2(\mathrm{n}=240)$ to record the $\mathrm{LV}$ volume, ejection fraction and mass, left atrial volume, and function parameters. The primary endpoints were a composite of cardiac death, myocardial infarction, and congestive cardiac failure. After a follow up of median 5.6 years, there was significant difference in left atrial functions with respect to mitral valve A velocity, LAVI and atrial fraction was found, while LA ejection fraction did not show significant difference.

In our study of chronic kidney disease patients with systolic dysfunction the left atrial volume index was ascertained to be a strong and only determinant of the diastolic dysfunction where as the other parameters of mitral inflow velocities and their ratio and the deceleration time could not achieve a significance.

\section{Limitations}

The study is limited by the small number of study population and by more than one operator echocardiography interrogation. 


\section{CONCLUSION}

Echocardiographic parameters as a stand alone tool for determining diastolic dysfunction in presence of chronic kidney disease are inadequate. Therefore, a combination of these parameters becomes mandatory to gauge the diastolic dysfunction in chronic kidney disease patients. Left atrial volume index may be proposed as a significant tool for diastolic dysfunction and it should be an essential part of echocardiographic interrogation in chronic kidney disease patients.

\section{REFERENCES}

[1] Prioli A, Marino P, Lanzoni L, et al. Increasing degrees of left ventricular filling impairment modulate left atrial function in humans. Am J Cardiol 1998;82(6):756-61.

[2] Paul B. Left atrial volume - a new index in echocardiography. J Assoc Physicians India 2009;57(6):463-5.
[3] Pearlman JD, Triulzi MO, King ME, et al. Left atrial dimensions in growth and development: normal limits for two-dimensional echocardiography. J Am Coll Cardiol 1990;16(5):1168-74.

[4] Cai QZ, Lu XZ, Lu Y, et al. Longitudinal changes of cardiac structure and function in CKD (CASCADE Study). J Am Soc Nephrol 2014;25(7):1599-608.

[5] Ersbøll M, Andersen MJ, Valeur N, et al. Early diastolic strain rate in relation to systolic and diastolic function and prognosis in acute myocardial infarction: a two dimensional speckle-tracking study. Eur Heart J 2014;35(10):648-56. http://www.r-project.org/.

[6] Hee L, Nguyen T, Whatmough M, et al. Left atrial volume and adverse cardiovascular outcomes in unselected patients with and without CKD. Clin J Am Soc Nephrol 2014;9(8):1369-76. 